\title{
FAUNA ASSOCIADA AO FITAL HALIMEDA OPUNTIA (LINNAEUS) LAMOUROUX (CHLOROPHYTA) DO RECIFE DA PONTA VERDE, MACEIÓ, ALAGOAS, BRASIL
}

\author{
Cardeluzia Guilherme dos Santos ${ }^{1}$ \\ Monica Dorigo Correia ${ }^{1}$
}

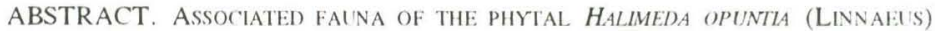
Lamouroux (Chlorophyta) on Ponta Verde reEF in Maceió, Alagoas. Brazil. The belt quadrat teonique were used to obtain five bimestral samples during 1992. Fifteen taxonomic groups were found and Amphipoda had numerical dominance. Polychaeta and Decapoda are also important taxa, principally in spring. All biomass aspects of algae are more important in winter.

KEY WORDS. Halimeda opuntia, phytal, associated fauna, Maceió, Brazil
\end{abstract}

No Brasil, os estudos sobre comunidades bentônicas associadas a algas foram realizados principalmente no litoral paulista, visando aspectos descritivos. A fauna séssil de Sargassum cymosum C. Agardh foi inicialmente caracterizada por Souza Lima (1969) na praia do Lamberto, Ubatuba. Para esta mesma alga, foi efetuada por MONTOUCHET (1972) a descrição da fauna vágil associada junto a enseada do Flamengo, Ubatuba. BOFFI (1972) caracterizou os aspectos ecológicos dos Ophiuroidea de titais das águas do Oceano Atlântico. A biologia de uma espécie de Isopoda foi descrita por PIRES (1975), junto ao fital Sargassum cymosum C. Agardh. Aspectos ecológicos do fital Halimeda opuntia (Linnaeus) Lamouroux foram apresentados por LEITE (1976), quando também realizou observações sobre a biologia do Amphipoda Hyale media (Dana) 1853. A fauna vágil de Sargaxsum cymosum C. Agardh foi comparada qualitativa e quantitativamente, com ênfase em Amphipoda por TARARAN (1977), nas praias do Lamberto e Grande, Uhatuba. As migrações de animais entre diferentes algas utilizadas como substrato em praias de Ubatuba foram caracterizadas por MONTOUCHET (1979). A carcinofauna do fital Halimeda opuntia (Linnaeus) Lamouroux e a variação sazonal da sua densidade foi demonstrada por GoUvÊA \& LEITE (1980). A alimentação e a distribuição do Amphipoda Hyale media (Dana) 1853 no fital da Práa do Poço, em Itanhaém, foram apresentadas por TARARAN (1980). A fauna móvel de Sargassum cymosum C. Agardh, em especial Gammaridae foi publicada por TARARAN \& WAKABARA (1981). Um estudo comparativo realizado por WAKABARA et al. (1983) caracterizou a fauna de Amphipoda presente no fital Sargassum cymosum C. Agardh, em duas praias de Itanhaém. Alguns aspectos

1) Laboratório de Ciências do Mar. Universidade Federal de Alagoas. 57022-970 Maceió. Alagoas. Brasil. 
LECENDA:

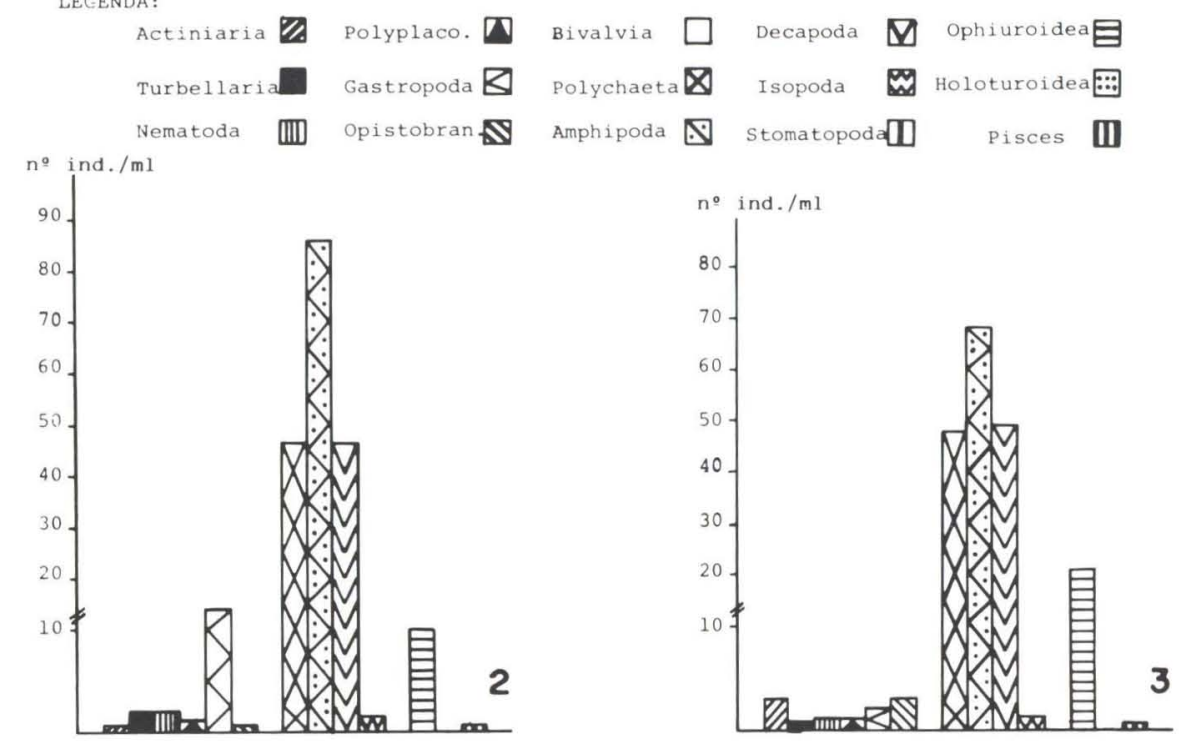

Figs 2-3. (2) Distribuição média dos táxons no verão; (3) distribuição média dos táxons no outono.

Período de outono. Foram consideradas as coletas realizadas nos meses de abril e maio. Para este período o número total dos organismos registrados foi de 1983 indivíduos/ml, tendo-se verificado 13 táxons zoológicos. Na variação média dos organismos encontrados, obteve-se como valor mínimo 0,4 indivíduos/ml para a Classe de Pisces e o máximo de 68,0indivíduos/ml para a Ordem Amphipoda. A Ordem Decapoda e a Classe Polychaeta também apresentaram importância quantitativa (Fig. 3).

Período de inverno. Optou-se por estudar as amostras obtidas nos meses de julho e agosto. Somando-se os dados encontrados para as amostras analisadas neste período, obteve-se um total de 2993 indivíduos/ml, incluídos em 14 grupos taxonômicos. As médias do presente período variaram entre a mínima de 0,2 indivíduos $/ \mathrm{ml}$ para a Classe Bivalvia e a máxima de 108,8 indivíduos $/ \mathrm{ml} \mathrm{com}$ relação a Ordem Amphipoda que foi o táxon dominante. Registrou-se também, a importância numérica da Ordem Zoanthidae e da Classe Polychaeta (Fig. 4).

Período de primavera. As amostras relacionadas a este período foram coletadas nos meses de outubro e novembro. Foi obtido para estas amostras um total de 4270 indivíduos $/ \mathrm{ml}$, os quais incluiram-se em 15 táxons. A média mínima encontrada foi de 0,1 indivíduos/ml verificada para os táxons Stomatopoda, Holuturoidea e Pisces, tendo-se caracterizado como a maior média, 204,8 indivíduos/ml para a Ordem Zoanthidae. Constatou-se, ainda, dados elevados referentes a Ordem Amphipoda e a Classe Polychaeta (Fig. 5).

2. BIOMASSA TOTAL

Com relação ao volume médio total encontrado junto às amostras da alga 

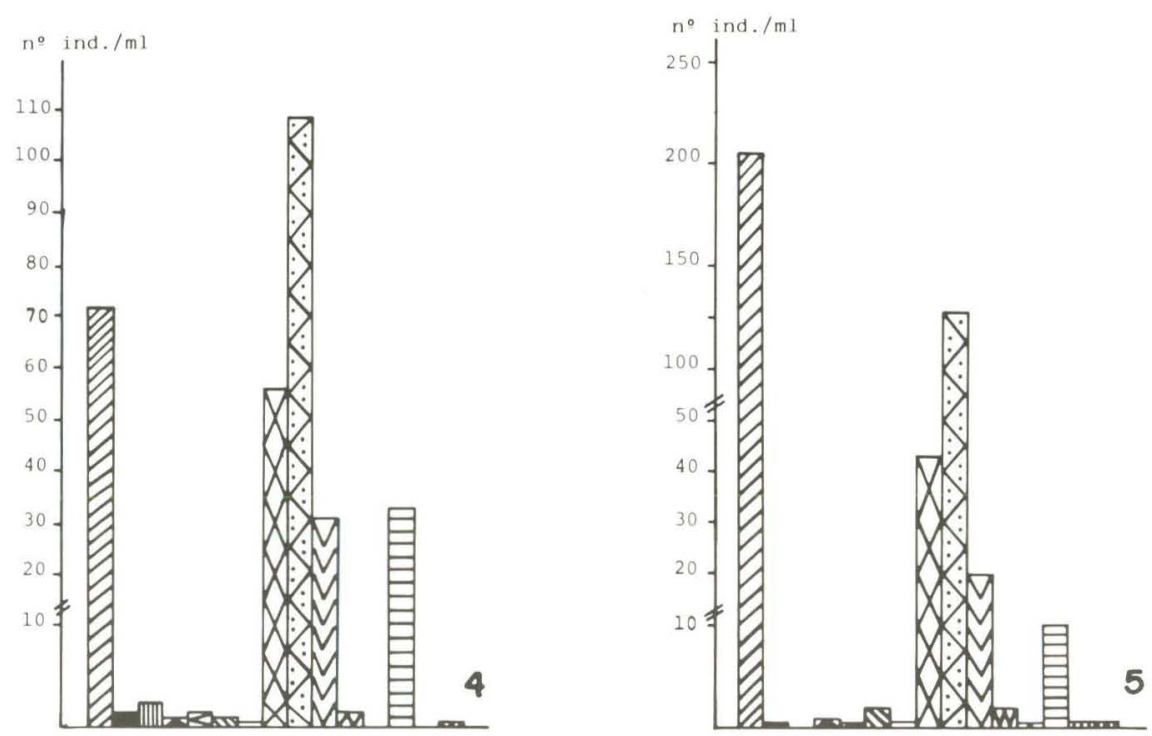

Figs 4-5. (4) Distribuição média dos táxons no inverno; (5) distribuição média dos táxons na primavera.

substrato, o valor mais elevado foi obtido para as amostras referentes ao período de inverno, quando registrou-se o volume de 390,3 ml. Correspondendo, consequentemente aos resultados obtidos para a biomassa. Desta forma, verificou-se para o período em questão a presença dos mais elevados pesos médios, tanto úmido com 538,7g, quanto seco, para o qual foram registrados $211,7 \mathrm{~g}$ (Fig. 6).

\section{ParAimetros ambientais}

Com relação aos dados obtidos para a temperatura do ar, verificou-se que a mais elevada ocorreu no mês de março e a mínima no mês de agosto. Entretanto, constatou-se para a temperatura da água o maior valor no mês de maio e o menor no mês de agosto.

Através das análises realizadas, obteve-se o maior valor de salinidade no mês de março e o menor no mês de agosto. Para os dados referentes ao oxigênio dissolvido, registrou-se a maior concentração para o mês de novembro e a menor para o mês de maio (Fig. 7).

\section{DISCUSSÃO}

Entre os táxons registrados, a Ordem Amphipoda foi a que apresentou em geral o maior número de indivíduos, sendo este táxon dominante numericamente na maioria das amostras analisadas. Estes resultados assemelham-se aos obtidos no litoral paranaense para o fital Pterocladia capillacea estudado por DUTRA (1985), como também no litoral paulista para o fital Sargassum cymosum caracterizado por TARARAN (1977) e TARARAN \& WAKABARA (1981), quando estes 
autores constataram a dominância numérica da Ordem Amphipoda. Entretanto, para alguns fitais do Recife de Piedade, litoral do Estado de Pernambuco, BragA (1983) verificou que o Gastropoda Tricolia affinis foi a espécie numericamente dominante.

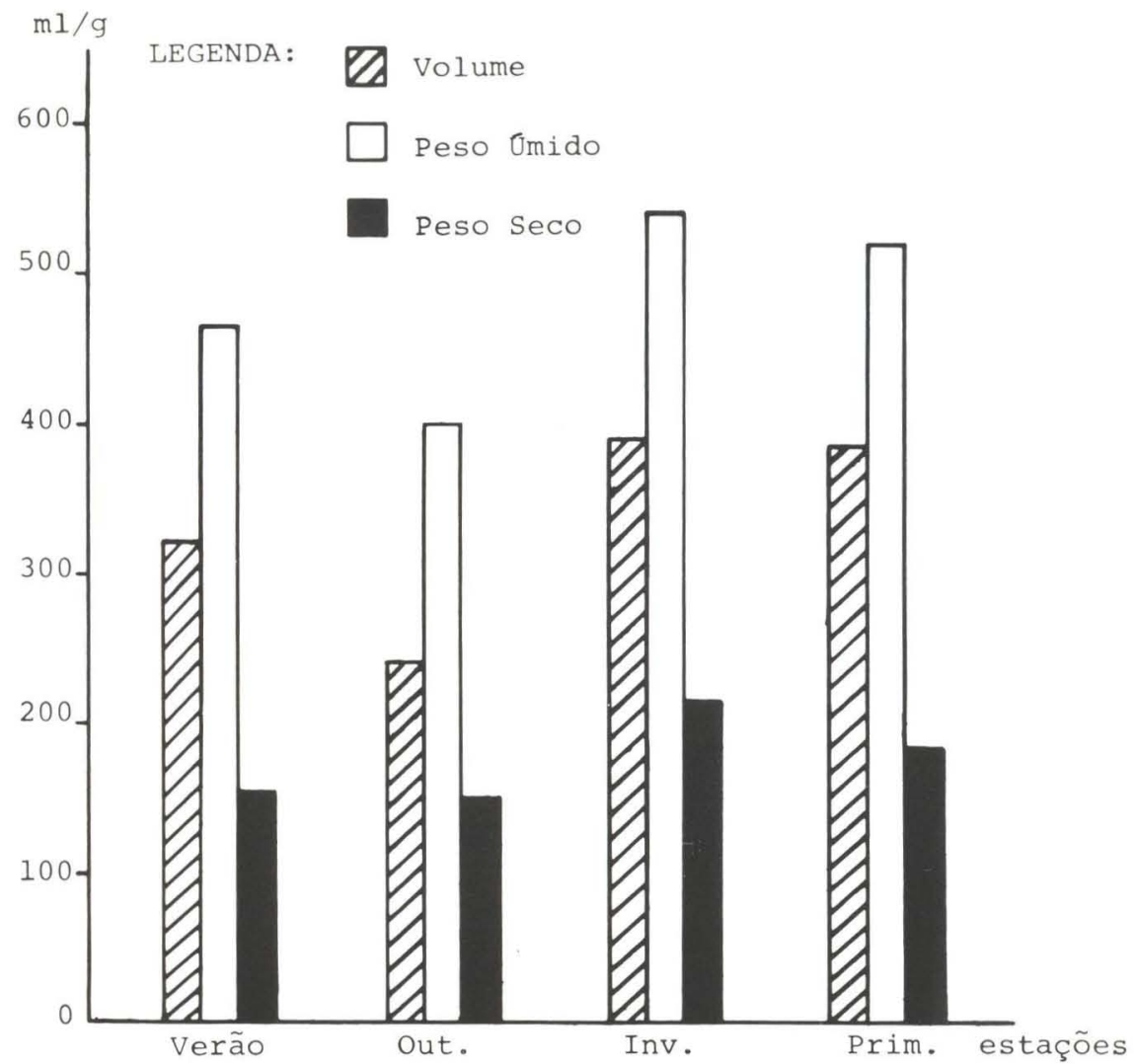

Fig. 6. Distribuição da biomassa ao longo das estações do ano.

No presente trabalho as Ordens Amphipoda e Decapoda, juntamente com a Classe Polychaeta, foram registradas em todas as amostras analisadas durante este estudo, tendo-se verificado o maior número de exemplares $/ \mathrm{ml}$ para o período de primavera. Estes dados corresponderam aos obtidos por SANTOS \& CORREIA (1992) que caracterizaram os fitais de seis gêneros de algas do recife da Ponta Verde, onde estes táxons também foram considerados bastante freqüentes para o fital Halimeda.

A presença da Classe Polychaeta foi considerada como frequente nas comunidades de fitais descritas por TARARAN (1977) e TARARAN \& WAKABARA (1981). No material analisado, verificou-se uma dominância numérica para a Classe Polychaeta nas amostras do mês de março, o qual correspondeu ao período 

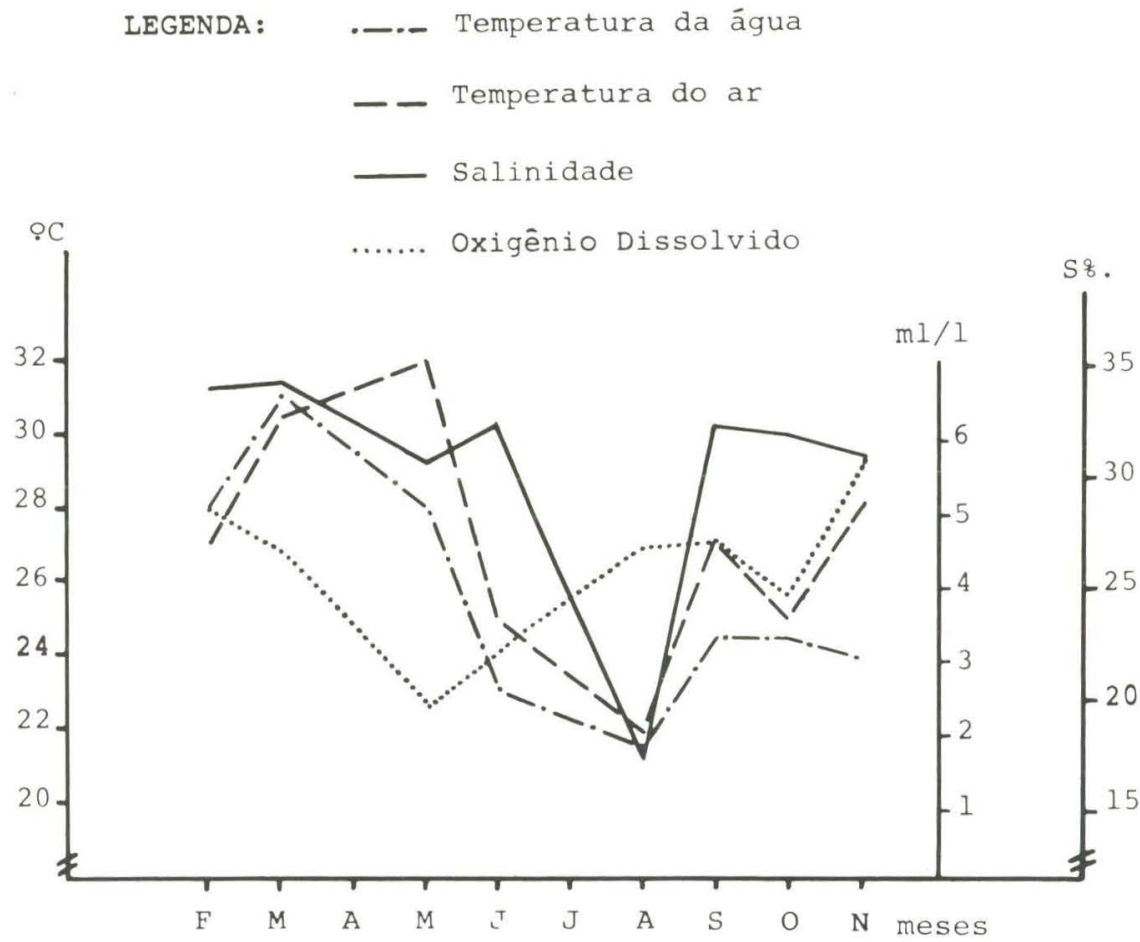

Fig. 7. Distribuição dos parâmetros ambientais ao longo dos meses.

de verão, quando ocorrem poucas chuvas no litoral nordeste do Brasil. Este fato, pode ser explicado por esta época corresponder ao período de recrutamento de Nereidae, táxon mais abundante dentro deste grupo zoológico.

CONNELl \& SOUZA (1983) demonstraram que a predação pode ser um fator primordial a ser considerado junto aos modelos de estrutura das comunidades, exceto onde exista outro mecanismo que reduza a ação deste fator. Nas amostras estudadas do fital Halimeda opuntia, cujas coletas foram realizadas na região entre-marés, provavelmente a ação dos parâmetros físicos e químicos foram os fatores determinantes na composição e distribuição dos organismos bentônicos, em função do estresse decorrente das oscilações das marés.

\section{CONCLUSÕES}

Com relação ao número total de organismos, verificou-se na primavera a maior concentração e diversidade de táxons. Constatou-se a dominância numérica da Ordem Amphipoda para 21 das 40 amostras analisadas neste estudo. Amphipoda, Decapoda e Polychaeta foram constantes em todas as amostras analisadas do fital Halimeda opuntia na região entre-marés do Recife da Ponta Verde, Maceió (Alagoas). A biomassa total mais elevadada da alga substrato foi registrada para o período de inverno, incluindo os três aspectos analisados. 
AGRADECIMENToS. À Profa Élika Guedes pela contirmaụào taxonônica da alg̨a suhstrato. À Profa Hilda Helena Sovierzoski pelo auxílio durante o trabalho de campo e pelas sugestoes no decorrer deste trabalho.

\section{REFERÊNCIAS BIBLIOGRÁFICAS}

Alves, M.S. 1991. Comunidade Faunística dos Prados da Fanerógama Halodule wrightii da Praia de Jaguaribe, Ilha de Itamaracá, Pernambuco. Tese de Mestrado, não publicada, Universidade Federal de Pernambuco, Recife, $139 \mathrm{p}$.

BoFFI, K.H. 1972. Ecological aspects of ophiuroids from phytal of S.W. Atlantic Ocean warm waters. Mar. Biol. 15 (4): 316-328.

Braga, R.A.P. 1983. Participação de Tricolia affinisis C.B. Adams, 1850 (Mollusca, Gastropoda) na comunidade dos animais vágeis associado as macroalgas dos arrecifes de Piedade (PE). An. Soc. Nordest. Zool. 1 (1): 80-81.

Connell, J.H. \& W. Sousa. 1983. On the evidence needed to judge ecological stability or persistence. Amer. Natur. 121 (6): 789-824.

Correia, M.D. 1991. Zonação Macrohentônica do Recife Coralíneo da Ponta Verde, Maceió - AL. Resumos do XVIII Congresso Brasileiro de Zoologia, Salvador, Resumo p.504.

Correia, M.D. \& H.H. Sovierzoski (no prelo). Levantamento da Macrofauna Bentônica Associada ao Recife Coralíneo da Ponta Verde, Maceió - AL. An. Soc. Nordest. Zool.

DHN 1992. Tábua das Marés. Diretoria de Hidrogratia e Naveg̨ação, Marinha do Brasil, 217p.

Dutra, R.R.C. 1985. A Fauna Vágil do Fital Pterocladia capillacea (Gmelin) Bornet \& Thuret (Rhodophyta, Gelidiaceae) da Ilha do Mel, Paranaguá, Paraná. Tese de Mestrado, não publicada, Universidade Federal do Paraná, Curitiba, 115 p.

GouvÊA, E.P. \& Y.M. LeITE. 1980. A carcinofauna do tital de Halimeda opuntia (Linnaeus) Lamouroux e a variação sazonal de sua densidade. Ciênc. Cult., São Paulo, 32 (6): 596-600.

LAB()REL-DEguen, F. 1963. Nota preliminar sobre a ecologia das pradarias de fanerógamas marinhas nas costas dos Estados de Pernambuco e da Paraía. Trab. Oceanogr. Fed. Pe. 3/4: 39-50.

LeITE, Y.M.R. 1976. Aspectos Ecológicos do Fital Halimeda opuntia (Linnaeus) Lamouroux. Tese de Mestrado, não publicada, Universidade de São Paulo, São Paulo, 47p.

Masunari, S. 1983 The phytal of the alga Amphiroa fiagilissima. Stud. Neotrop. Fauna Environ. 18 (3): 151-162.

- 1984. Organismos do fital Amphiroa beavoisii Lamouroux, 1816

(Rhodophyta: Corallinaceae). I - Autoecologia. Bol. Zool. 7: 57-148.

Montouchet, J.P. 1972. A Fauna Vágil Associada a Sargassum cymosum C. 
Agardh, na Enseada do Flamengo, Uhatuba, SP. Tese de Doutorado, não publicada, Universidade de Sào Paulo, São Paulo, 72p.

1979. Sur la communatuté des animaux vagiles associés à Sargecsisum cymosum C. Aggardh, à Uhatuba, Etat de São Patulo, Brésil. St. Neotrop. Fauma Environ. 14: 33-64.

PIRES, A.M.S. 1975. Sobre a Biologia de Janaira gracilis (Crustacea, Isopoda, Ascellota) da Fauna Vágil de Sargassum cymosum. Tese de Mestrado, não publicada, Universidade de São Paulo, São Paulo, 51 p.

Santos, C.G. \& M.D. Correia. 1992. Fitals do Recife Coralíneo da Ponta Verde. Mateió - AL. Resumos do XIX Congresso Brasileiro de Zoologia. Belém, p. 14.

Souza Lima, H. 1969. Fauna Séssil do Sargassum cymosum da Praia do

Lamberto (Estado de São Paulo): Composição e Considerações sobre a Localização das Espécies na Planta. Tese de Mestrado, não publicatda. Universidade de Säo Paulo, São Paulo, 27p.

STRICKLAND, J.D.H. \& T.R. PARSUNS. 1972. A practical handhork of seawater analysis. Bull. Fish. Res. Bd. Can. 167: 1-310.

Tararan. A.S. 1977. A Fauma Vágil de Sargassum cymosum C. Agardh, 1820 da Praia do Lamberto e Praia Grande, Ubatuba, São Paulo, com especial referência ans Gammaridae (Crustacea. Amphipoda). Tese de Mestrado, não publicadda, Universidade de São Paulo, São Paulo, 73p.

1980. Alimentação e Distribuição de Hyale media (Crustacea, Amphipoda) do Fital da Praia do Poço, Itanhaém (SP), com Olsservaçōes sobre a Predação da Espécie por Alguns Peixes da Região. Tese de Doutorado, não publicada, Universidade de São Paulo, São Paulo, 187p.

TARARAN. A.S. \& Y. Wakabara. 1981. The mohile fauna especially Gammaridea of Sargassum cymosum. Mar. Ecol. Progress Series 5: 157-163.

TARARAN, A.S.; Y. WAKABARA \& F.P.P. LeITE. 1986. Vertical distrihution of Amphipods living on algate of hrasilian intertidal rocky shore. Crustaceana 51 (2): 183-187.

Wakabara, Y.: A.S. TARaran \& H.M. Takeda. 1983. Comparative study of the amphipod fauna living on Sargessum of two Itanhaém shores. Brazil. J. Crust. Biol. 3 (4): 602-607. 